\title{
Identification of two novel elements involved in human MUCl gene expression in vivo
}

\author{
Toshiyuki Shiraga, ${ }^{1^{*}}$ David Smith, ${ }^{1,2^{*}}$ Hugh N Nuthall, ${ }^{1 * *}$ Michael A Hollingsworth, ${ }^{2}$ and Ann Harris ${ }^{1}$ \\ ${ }^{1}$ Paediatric Molecular Genetics, Institute of Molecular Medicine, University of Oxford, John Radcliffe \\ Hospital, Oxford, UK \\ ${ }^{2}$ Eppley Institute, University of Nebraska Medical Center, Omaha, NE, USA \\ Accepted January 10, 2002
}

\begin{abstract}
Background: MUC1, a membrane-tethered glycoprotein that is expressed on a number of epithelial cell types in vivo, is over-expressed in adenocarcinomas and thought to play a significant role in tumour progression and metastasis. Hence, elucidation of the mechanisms of regulation of $M U C 1$ gene expression is of considerable biological importance. Our aim was to evaluate regulation of $M U C 1$ expression in vivo.

Materials and Methods: DNase I hypersensitive sites (DHS) were mapped in chromatin from human cell lines and human MUC1 transgenic mice. MUC1 expression was evaluated by RT-PCR and Northern blots.

Results: We identified two novel DHS in the MUC1 promoter at $-750 \mathrm{bp}$ and $-250 \mathrm{bp}$ from the transcriptional start site. These DHS were detected in human cell lines

and in a human MUC1 transgene in mice. The -750 DHS was apparent in many cell types irrespective of the level of MUC1 expression but the -250 DHS was only evident in cells that express $M U C 1$ and its intensity correlated with the abundance of MUC1 transcripts. The -250 DHS became undetectable in cell lines representing a transition from colon adenoma to carcinoma, commensurate with a significant reduction in $M U C 1$ expression.

Conclusions: The -750 and -250 regions are conserved between the human MUC1 and mouse Mucl genes and may be associated with functionally important genetic elements. The DHS at $-\mathbf{2 5 0}$ is in the vicinity of previously defined purine/pyrimidine mirror repeat elements that may form intramolecular H-DNA structures, which can alter the accessibility of chromatin to regulatory proteins.
\end{abstract}

\section{Introduction}

MUCl is a cell surface-associated mucin glycoprotein that is highly overexpressed and differentially glycosylated by various adenocarcinomas (1). The MUCl protein plays a role in the biological properties of tumour progression, especially the process of metastasis.

The promoter of the MUCl gene has been partially characterized and some cis elements that are important for basal promoter activity have been identified (2-4). Sequential deletions, specific deletions and site specific mutations of sequences $5^{\prime}$ to the $M U C 1$ gene revealed that at least $600 \mathrm{bp}$ of upstream sequence was required for maximal promoter activity in transient transfections $(2,3)$. This effect may be partly due to deletion of an Spl site located approximately $-570 \mathrm{bp}$ from the transcription start site, an AP-3 site adjacent to this, or several other potential cis elements just downstream. DNase I footprint and/or gel shift analysis revealed several putative elements in the $5^{\prime}$ region that may be

Address correspondence and reprint requests to: Ann Harris, Paediatric Molecular Genetics, Institute of Molecular Medicine, University of Oxford, John Radcliffe Hospital, Oxford, OX3 9DS, UK; fax: 44-1865-222626; e-mail: aharris@molbiol.ox.ac.uk.

* Contributed equally.

** Current address, Montreal Neurological Institute. bound by protein factors. Based on these results, two different regions have been postulated to be involved in the basal control of expression of the MUC1 gene. One element, E-MUC1, located immediately upstream of the TATA box, within $100 \mathrm{bp}$ of the transcription start site, may bind specific transcription-regulating factors responsible for determining the tissue-specific expression of the $M U C 1$ gene (3). A second region between -485 and -505 appears to be involved in the regulation of transcription of the $M U C 1$ gene (2). This DNA sequence binds to a $45 \mathrm{kDa}$ protein present in breast carcinoma cell line, MCF7, and acts in an orientationdependent fashion in reporter constructs. In addition, a secreted factor capable of stimulating the production of MUCl by human colon carcinoma cells has been described (5). This protein, called mucomodulin, has an apparent molecular mass of $70 \mathrm{kDa}(4)$ and was observed to stimulate the production of MUCl in greater than $70 \%$ of colon carcinoma cell lines tested. A subsequent study identified a cis element, called RME, in the flanking $5^{\prime}$ region of the MUC1 gene that renders CAT constructs responsive to treatment with mucomodulin (4). The Spl transcription factor has also been implicated in cell type specific transcription of $M U C 1$ through several binding sites in the promoter $(3,6)$. Further, $M u c l$ expression has been shown be hormonally regulated (7). 
The regulatory mechanisms that result in overexpression of $\mathrm{MUCl}$ in tumours have not been established. Activation of Signal Transducer and Activator of Transcription (STATs) proteins are probably important because the $M U C 1$ promoter contains a functional STAT3/ 1 element that is responsive to IL- 6 and $\gamma$-interferon in reporter gene assays (8). In addition, MUC1 expression in human mammary cell lines is regulated by the c-ErbB2 and ras signalling pathways (9).

Our aim was to search in vivo for novel regulatory elements within the $M U C 1$ gene and its $5^{\prime}$ and $3^{\prime}$ flanking regions by investigating DNase I hypersensitive sites (DHS), which are often associated with these elements. DHS were evaluated in human cell lines that express or do not express the gene, in a colonic cell line showing progression from adenoma to carcinoma (10) and in transgenic mice carrying a human MUC1 genomic construct. The transgenic mice have a $10.6 \mathrm{~kb}$ Sac II fragment of genomic DNA that includes $1.6 \mathrm{~kb}$ of upstream sequence and $1.9 \mathrm{~kb}$ of downstream sequence (11) and show tissue-specific expression of MUCl similar to that seen in humans (12).

We defined two novel DNase I hypersensitive sites in the MUC1 promoter. These two DHS are detected in vivo in cell lines and in certain tissues from $M U C 1$ transgenic mice that express the human transgene. Further, the appearance of one of these DHS correlates with high levels of MUC1 transcription in certain carcinoma cell lines. This DHS may be associated with a regulatory element that causes elevated $M U C 1$ transcription in some primary cell types and tumour cell lines.

\section{Materials and Methods}

\section{Cell Culture}

The following cell lines were used; $\operatorname{HPAF}(13), \mathrm{Caco} 2$ (14), HT29 (15) and MCF7 (16) were cultured in DMEM; the lymphoblastoid cell line 37566 was cultured in RPMI 1640 and the AA/Cl and AA/Cl/ SBI0C colonic cell lines in DMEM supplemented with $1 \mu \mathrm{g} / \mathrm{ml}$ hydrocortisone and 0.2 units $/ \mathrm{ml}$ insu$\operatorname{lin}(10)$.

\section{Transgenic Mice}

The generation of C57/BL6 mice carrying the $10.6 \mathrm{kB}$ Sac II fragment of the human MUC1 gene are described elsewhere $(11,12)$. Control mice were wild type C57/BL6. Mouse tissues were collected immediately after death and either placed in liquid nitrogen for RNA extraction or processed directly for chromatin extraction.

\section{Extraction of Chromatin}

Chromatin was extracted from transgenic and normal mouse tissues and from cell lines as described previously (17-19). For all tissues chromatin was extracted from the whole organ without further microdissection.
RNA Extraction, Reverse Transcriptase-PCR (RT-PCR) and Northern Blots

Total RNA was extracted from transgenic and normal mouse tissues and from cell lines by standard methods (20). All tissues were evaluated for transcription of human $M U C 1$ and mouse $M u c 1$ mRNA by standard methods of RT-PCR (Superscript). PCR parameters were $95^{\circ} \mathrm{C} 1 \mathrm{~min}, 50^{\circ} \mathrm{C} 2 \mathrm{~min}, 72^{\circ} \mathrm{C} 5 \mathrm{~min}$ for 30 cycles. The locations of primers used for RT-PCR were MUCIHMA 5' ACTACTACCAAGAGCTG 3' (J05582: 3264-3280; M84683: 1339-1355) and MUCIHMB 5' CTCATAGGATGGTAGGT3' (J05582: 3693-3677; M84683: 1762-1746). The 429 bp human cDNA product is cleaved by Dra III into 273 and $156 \mathrm{bp}$ fragments and the $424 \mathrm{bp}$ murine cDNA product by Eag I into 261 and $163 \mathrm{bp}$ fragments. These data are not truly quantitative as the MUCIHMA primer, though matching the human MUCl sequence exactly has a 1 base mismatch with the murine gene. The MUC1HMB primers are $100 \%$ matched to human and mouse gene sequences. The $\beta$-actin primers were $\beta$ 3' ATGCCATCCTGCGTCTGGACCTGGC and $\beta 5^{\prime}$ AGCATTTGCGGTGCGACATGGAGGG producing a $607 \mathrm{bp}$ fragment from mouse RNA. RT-PCR for $M U C 1$ expression from human cell lines was carried out as described previously (21). Northern blots of total RNA from each cell line were carried out by standard methods and were probed with 5'MUCl probe described below.

\section{DNase I Hypersensitivity Assays}

Chromatin from mouse tissues and cell lines was probed for DNase I hypersensitive regions by standard methods (22). The probes used for DHS Southern blots were 5'MUCl (bases 1-345 of the MUC1 cDNA), PB352 a 352 base pair Pvu I/BamH I fragment of the MUC1 CDNA (J05582: 3748-4100), BS308 (BamH I/Sac I M61170: 6633-6941), AB350 (Afl II/Bsm I M61170: 3255-3600) and BS280 (BspLU11 I/Sac I U16175: 4675-4953). AB350 hybridized to human genomic DNA only and not to mouse genomic DNA under the conditions used and BS280 hybridized to mouse but not human DNA.

\section{Results}

A number of previous studies have been carried out on the MUC1 promoter in vitro. Important sequence motifs identified by the previous studies in the $5^{\prime}$ region of the $M U C 1$ gene are shown in Fig. 1.

\section{Identification of DHS in the MUC1 Gene in Human Cell Lines}

The $M U C 1$ coding region is flanked by BamH I sites at -2239 (M61170: 632) with respect to the transcriptional start site and within the 3' untranslated region (M61170:6633). This BamH I fragment of about $8 \mathrm{~kb}$ (the sequence of M61170 predicts a $6 \mathrm{~kb} \mathrm{BamH} \mathrm{I}$ fragment due to polymorphisms in the length of the MUC1 tandem repeat) and the adjacent 3'Bam $\mathrm{H} \mathrm{I}$ 


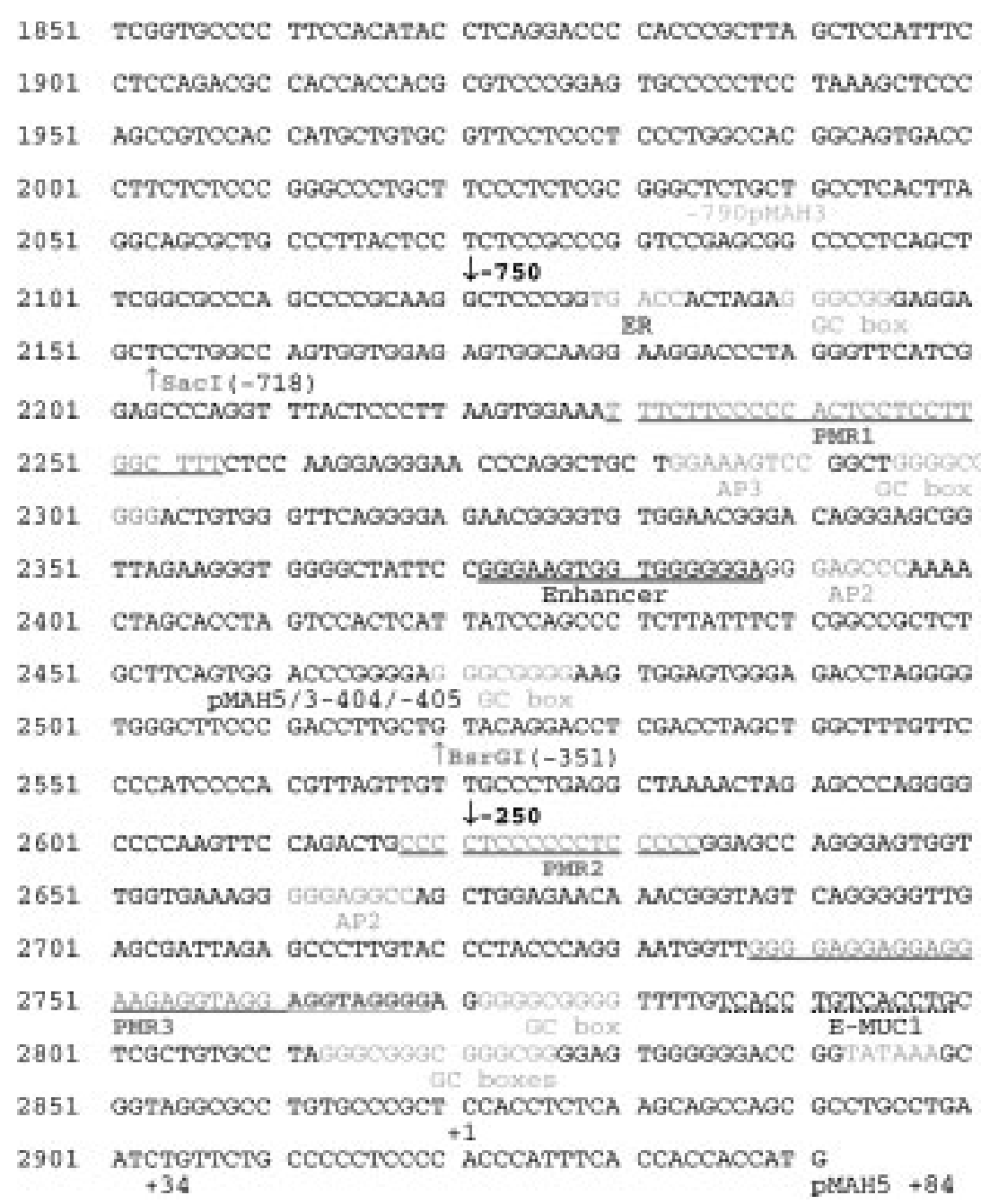

Fig. 1. The sequence of the immediate $5^{\prime}$ promoter region of the human MUC1 gene. (from X69118) Important sequence motifs previously described by others and the novel DHS are shown. fragment were probed for DHS using the $5^{\prime} \mathrm{MUCl}$, PB352 and BS308 probes shown in Figure 2A. Two DHS were identified in the $5^{\prime}$ promoter region of MUCl in chromatin from the HPAF pancreatic adenocarcinoma cell line. Figure $2 \mathrm{~B}$ shows an $8 \mathrm{~kb}$ $B a m \mathrm{H}$ I genomic fragment hybridising to the $5^{\prime} \mathrm{MUCl}$ probe and subfragments at 6.5 and $6 \mathrm{~kb}$ that correspond to DHS at about $-750 \mathrm{bp}$ and $-250 \mathrm{bp}$ with respect to the transcriptional start site. BsrG I (x69118: 2520) and Sac I (x69118: 2154) cleavage sites at -351 and -717 respectively from the transcription start site enable confirmation of the approximate location of the DHS by the BamH I/Sac I and BamH I/BsrG I double digestion (Fig. 2). These DHS were also seen with the PB352 probe. Screening of DNA lying $3^{\prime}$ to the BamH I site at M61170:6633 failed to reveal any additional DHS in HPAF or lymphoblastoid (37566) cell line chromatin.

Additional cell lines were evaluated to establish whether the -750 and -250 DHS were seen only in chromatin from the HPAF pancreatic adenocarcinoma or were a more general phenomenon. Unlike in HPAF where only 1 genomic Bam $\mathrm{H}$ I fragment hybridises to the 5'MUCl probe, as the cell line is homozygous or hemizygous for MUCl (Fig. 3A), all other cell lines analysed showed 2 genomic fragments presumably due to variation in the MUC1 tandem repeat number on the 2 alleles. Chromatin isolated from the breast carcinoma cell line MCF7 contained the same two DHS though at a reduced intensity (Fig. 3B). The -750 bp site was also seen in the lymphoblastoid cell line (37566) (Fig. 3C) and the colon carcinoma cell line HT29 (data not shown).

Evaluation of the -750 and -250 DHS in a Colon Adenoma to Carcinoma Transition

The AA/Cl (adenoma) and AA/C1/SB10C (carcinoma) cell lines represent models of cancer progression in the human colon (10). The AA/Cl line is a premalignant variant of a human colonic adenoma cell line PC/AA derived from a familial polyposis coli patient. The AA/C1/SB10C line was generated by treating $\mathrm{AA} / \mathrm{Cl}$ with agents that induce differentiation and carcinogenesis. Though MUCl is expressed at a low levels in normal human colonic 

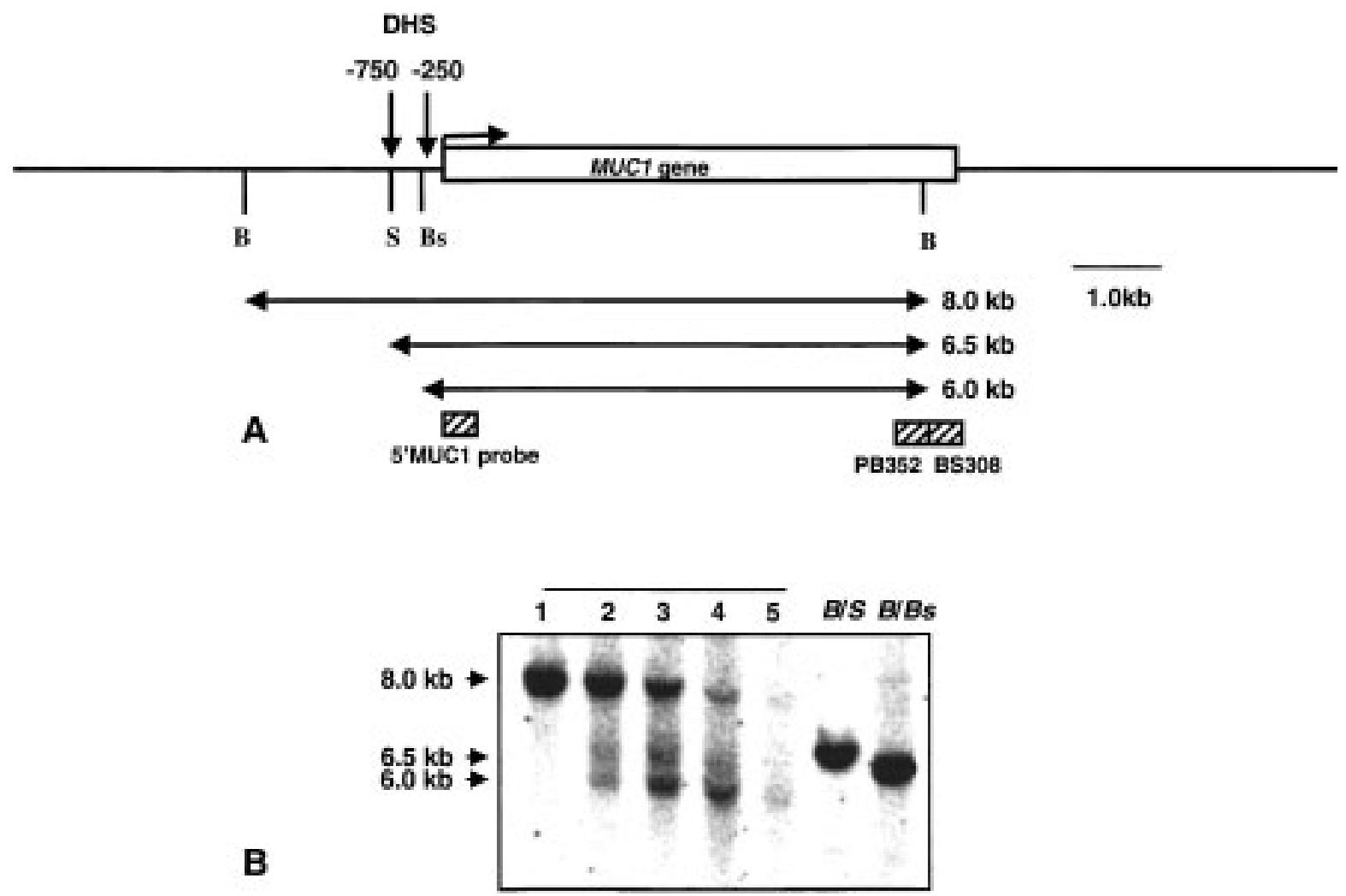

Fig. 2. DNase I hypersensitive sites at $-750 \mathrm{bp}$ and $-250 \mathrm{bp}$ with respect to the transcriptional start site of the human MUC1 gene in HPAF chromatin. (A) Map of region. Arrows above the line denote the DHS positions. Restriction sites shown below the line are B = BamH I, Bs = BsrG I, S = Sac I. Below the line are shown the probes (hatched) and the subfragments produced by DHS. (B) Southern blot of DNase I digested HPAF chromatin cleaved with BamH I and hybridised with 5'MUCl probe. Lanes $1\left(0^{\circ} \mathrm{C}\right)$ and $2\left(37^{\circ} \mathrm{C}\right)$ show DNA prepared from nuclei which had not been treated with DNase I. Lanes 3-5 show DNA prepared from nuclei treated with increasing amounts of DNase I; (Lane 3, 20 units; Lane 4, 40 units; Lane 5, 80 units DNase I). The detection of the DHS in lane 2 is probably due to endogenous DNase I activity at $37^{\circ} \mathrm{C}$.

epithelium and colon carcinoma-derived cell lines $(23,24)$ these 2 cell lines have been shown to express MUCl glycoprotein (25). Evaluation of the -750 and -250 DHS in these 2 cell lines showed both DHS in the adenoma (AA/Cl) (Fig. 3D) while only the -750 DHS was evident in the carcinoma cell line (AA/C1/SB10C) (Fig. 3E).

\section{Expression of the Human MUC1 Gene in Human Cell Lines}

There have been many reports on the relative expression levels of MUCl mRNA in different cell lines. To evaluate the potential significance of our data on the presence of the -750 and -250 DHS all cell lines were tested by a semi-quantitative RT-PCR assay for MUC1 and by northern analysis. Fig. 4A shows RT-PCR data for MUCl mRNA expression (656 bp product) relative to a housekeeping gene $\beta$ glucocerebrosidase (572 bp product). As expected high levels of MUCl mRNA are seen in the HPAF line and MCF7 while the MUCl gene expression is barely detectable in the lymphoblastoid cell line (37566). MUC1 expression in the $\mathrm{AA} / \mathrm{Cl}$ cell line is significantly less than in HPAF and further reduced in the AA/C1/SB 10C cell line.
These data were confirmed and extended by northern analysis (Fig. 4B), which showed that the levels of MUCl mRNA in HPAF are significantly higher than in MCF7, and that MUCl mRNA was undetectable in the 37566, $\mathrm{AA} / \mathrm{Cl}$ and $\mathrm{AA} / \mathrm{Cl} /$ SB 10C cell lines.

Presence of the -750 and -250 DHS Correlates with MUC1 Expression

The -750 DHS was weakly evident in all cell lines analysed irrespective of $M U C 1$ expression levels. In contrast, presence of the -250 DHS correlated with MUC1 expression levels. The -250 DHS was not detected in the lymphoblastoid cell line (37566) in which very low levels of MUCl mRNA were seen by RT-PCR. In MCF7, which expresses moderate amounts of MUC1 mRNA, the -750 and -250 DHS are of approximately equal intensity. In HPAF, which expresses very high levels of MUCI mRNA the -250 DHS is of much greater intensity than the -750 DHS. In cells representing the adenoma (AA/C1) to carcinoma (AA/C1/SB 10C) transition, which is accompanied by a decrease in MUCl mRNA, the -250 DHS disappears. 

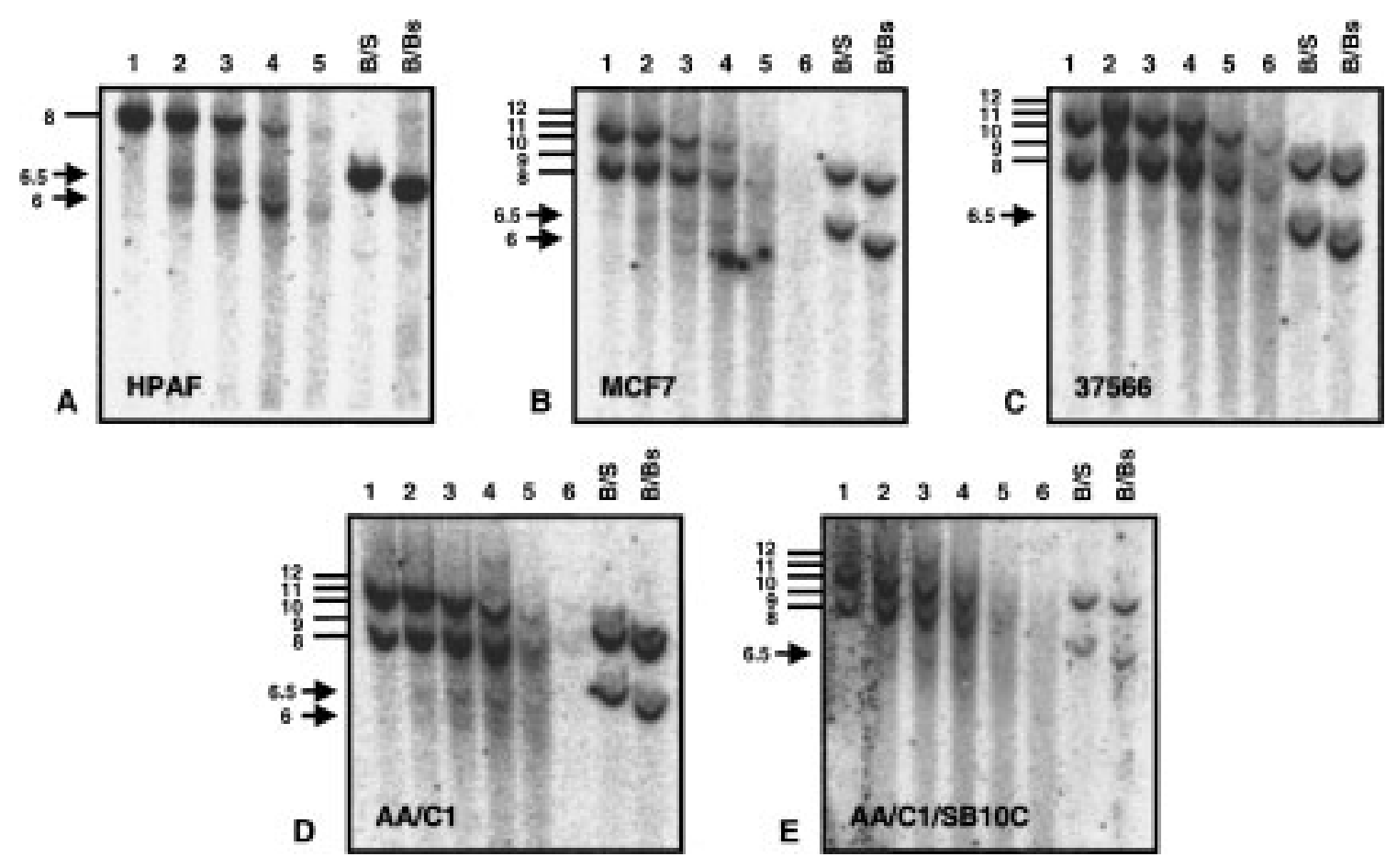

Fig. 3. Detection of the $\mathbf{- 7 5 0}$ and $\mathbf{- 2 5 0}$ DHS in chromatin from cell lines. Southern blot of DNase I digested chromatin from (A) HPAF, (B) MCF7, (C) lymphoblastoid (37566), (D) AA/C1, (E) AA/C1/SB 10C cleaved with BamH I and hybridised with 5'MUC1 probe. Lanes $1\left(0^{\circ} \mathrm{C}\right)$ and $2\left(37^{\circ} \mathrm{C}\right)$ show DNA prepared from nuclei which had not been treated with DNase I. Lanes 3-6 (Lanes 3-5 in A) show DNA prepared from nuclei treated with increasing amounts of DNase I; (lane 3, 20 units; Lane 4, 40 units; Lane 5, 80 units, Lane 6, 160 units DNase I). Lanes marked B/S and B/Bs denote double digests of the samples in lane 1 of each panel to map the DHS. B = BamH I, Bs = BsrG I, S = Sac I. DHS are seen at $6.5 \mathrm{~kb}$ in all panels and in A, B and D also at $6 \mathrm{~kb}$.

\section{Identification of DHS in the Human MUC1 Gene in Transgenic Mice}

Chromatin was extracted from tissues of the MUC1 transgenic mice and evaluated for DHS in the human transgene. Figure 5 shows DNase I treated chromatin from transgenic mouse stomach, lung and kidney. A probe specific for human $M U C 1$ (within intron 1 and part of exon 2 of the gene) hybridised to $3 \mathrm{BamH} \mathrm{I}$ fragments in transgenic mice carrying the $10.6 \mathrm{~kb}$ Sac II fragment of human MUC1 (Fig. 5A,C,E) instead of the expected single fragment. This suggests there are 2 or more copies of the human gene integrated into these mice. In contrast, a probe specific for the murine mucl gene (also within intron 1 and part of exon 2 of the murine gene) hybridised to a single Bam $\mathrm{H}$ I fragment of about $12 \mathrm{~kb}$, as expected (Fig. 5B,D,F). The human-specific probe used in Fig. 5 is predicted to hybridise to a BamH I fragment of about $9.2 \mathrm{~kb}$ based on the restriction fragment map of the human MUC1 $10.6 \mathrm{~kb}$ Sac II fragment (11). This is likely to be the smallest of the Bam $\mathrm{H}$ I fragments, allowing for slight variation between the migration of $1 \mathrm{~kb}$ ladder molecular weight markers and DNase I treated, restriction enzyme digested chromatin. Figure 5A,C,E show appearance of the -750 and -250 DHS as 6.5 and $6 \mathrm{~kb}$ subfragments of the human $M U C 1$ genomic fragments. Different DHS (detected as 5.6, 5.0 and $4.6 \mathrm{~kb}$ subfragments) are seen with the mouse specific probe, hybridising to the murine $\mathrm{Mucl}$ gene as shown in panel B. (The mouse-specific DHS are not evident in chromatin from lung and kidney in which the murine $\mathrm{Mucl}$ gene is expressed, possibly due to insufficient sensitivity to detect chromatin changes in only a small percentage of cells in the tissue sample.) The -250 DHS is more evident than that at -750 bp (Fig. 5A) in chromatin from transgenic mouse stomach. The two DHS are of equal intensity in lung chromatin (Fig. 5C) and in kidney (Fig. 5E). Both DHS were also seen in small intestine and pancreas at low intensity (data not shown).

\section{Expression of the Human MUC1 Gene in Transgenic Mice}

Figure 6 shows expression of the $M U C 1$ transgene and endogenous mucl using RT-PCR on RNA from transgenic mice. The primers MUCIHMA and MUC1HMB amplify a fragment of $429 \mathrm{bp}$ from the human gene that is cleaved by Dra III into (fragments of 273 and $156 \mathrm{bp}$ ) and $424 \mathrm{bp}$ from the murine gene that is cleaved by Eag I into 261 and $163 \mathrm{bp}$ fragments. Cleavage of the RT-PCR product with the appropriate enzyme enables discrimination of the human and mouse cDNAs. The assay is not truly quantitative as the PCR reactions are likely to be of unequal efficiency due to a 1 base pair mismatch with the mouse sequence in the 


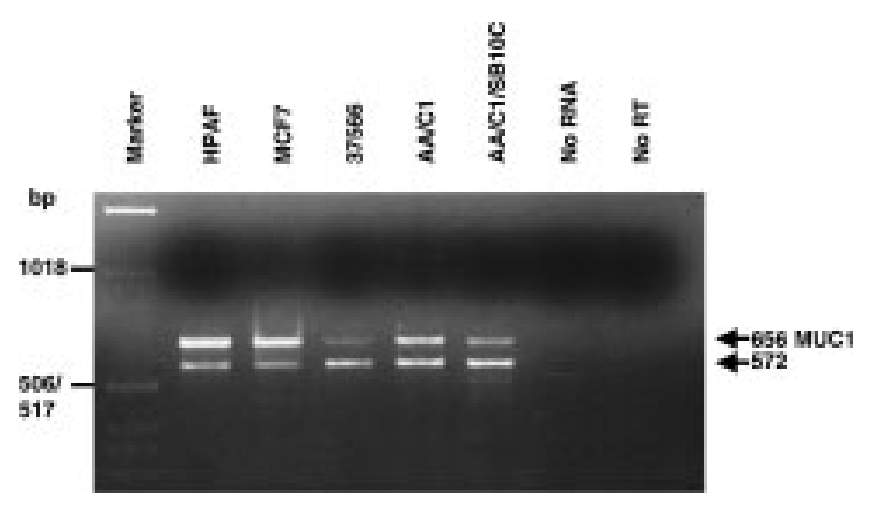

A

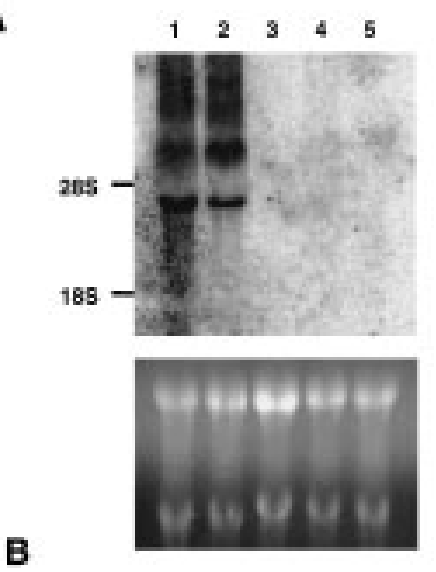

Fig. 4. Expression of $M U C 1$ in the cell lines analysed in figure 3. (A) RT-PCR using primers for $M U C 1$ that produce a $656 \mathrm{bp}$ fragment and for $\beta$ glucocerebrosidase that produce a 572 bp fragment. (B) Northern blot of RNA from cell lines, probed with 5'MUCl.

MUCIHMA primer. However, expression of the human MUCl gene is seen in lung, stomach, pancreas and kidney (Fig. 6), and also in small intestine, bladder, mammary gland and ovary (data not shown).

\section{Comparison of the Human MUC1 and Murine mucl Gene Promoters}

The 5' untranslated (X69118: 1851-2941) region of MUC1 shows approximately $74 \%$ identity between human and mouse. Of particular interest are the regions shown in Fig. 7 which correspond to the A) DHS -750 and B) DHS -250 . There is high homology between the human and mouse genes immediately $3^{\prime}$ to the -750 DHS which may reflect functional conservation in this region. The $-250 \mathrm{DHS}$ region is flanked by regions of high human/mouse sequence conservation both $5^{\prime}$ and $3^{\prime}$ to the site. Since mapping of DHS by Southern blotting is not exact, even with restriction enzyme sites to confirm the locations, these regions of high homology between the human and mouse genes may represent functional importance.
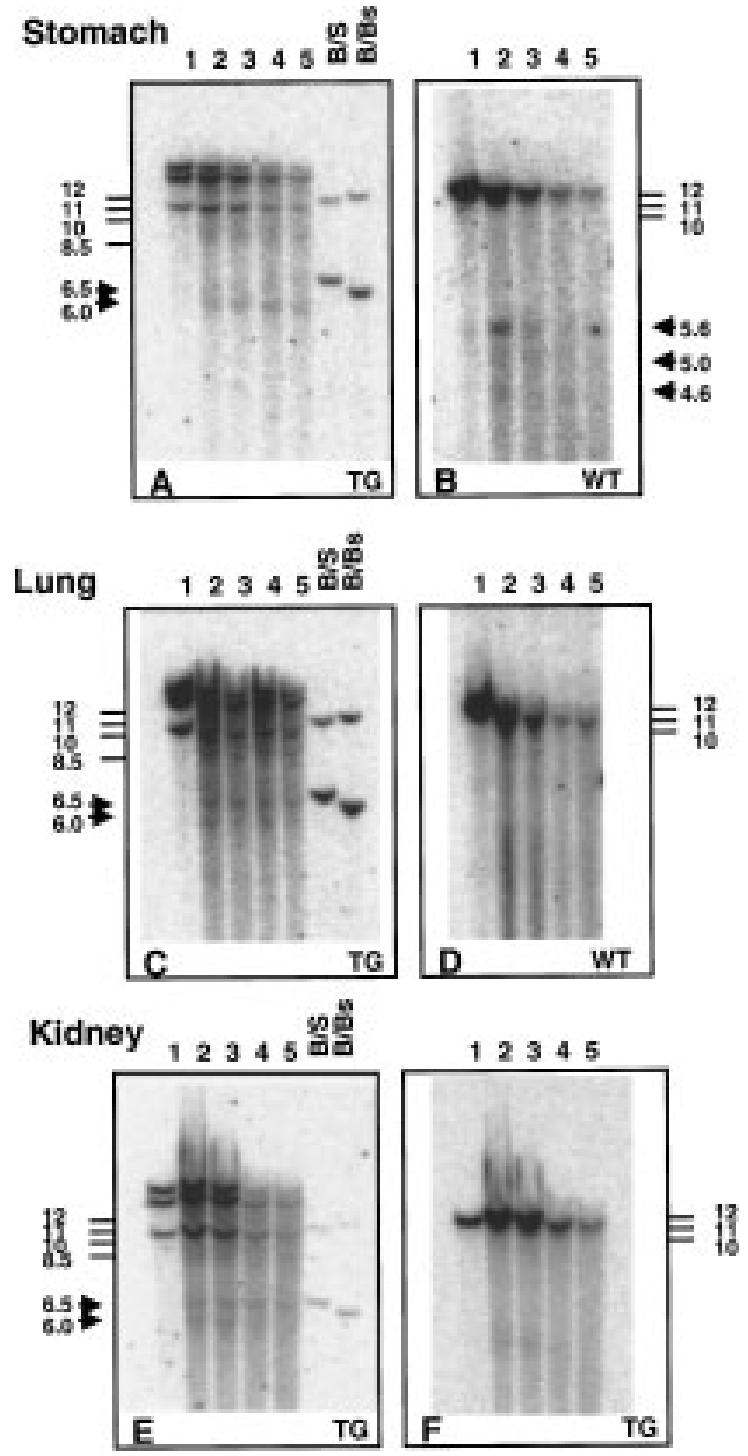

Fig. 5. Detection of the $-750 \mathrm{bp}$ and $-250 \mathrm{bp}$ DNase I hypersensitive sites in MUC1 transgenic mice. Southern blots of DNase I digested chromatin from transgenic mouse (TG) or wild type (WT) tissue samples digested with BamH I and hybridised with human specific (A, C, E) and mouse specific (B, D, F) $M U C 1 / m u c 1$ probes. For each panel, Lane 1 shows DNA prepared from nuclei which had not been treated with DNase I. Lanes 2-5 show DNA prepared from nuclei treated with a constant amount of DNase I (40U) for increasing lengths of time, 2, 2 min; 3, 5 min; 4, 10 min; 5, 15 minutes. The human-specific probe detects 3 genomic fragments and DHS at 6.5 and $6 \mathrm{~kb}$ in chromatin from stomach (A) lung (C) and kidney (E). The mouse specific-probe detects a genomic fragment of about $12 \mathrm{~kb}$ and DHS in stomach only (B) at 5.6, 5.0 and $4.6 \mathrm{~kb}$ but not in lung (D) or kidney (F).

\section{Discussion}

DNase I hypersensitive sites in chromatin are often associated with regulatory elements and so can be informative in the identification of control elements that are active in vivo. Regulation of expression of the MUCl gene has been studied by in vitro methods; 

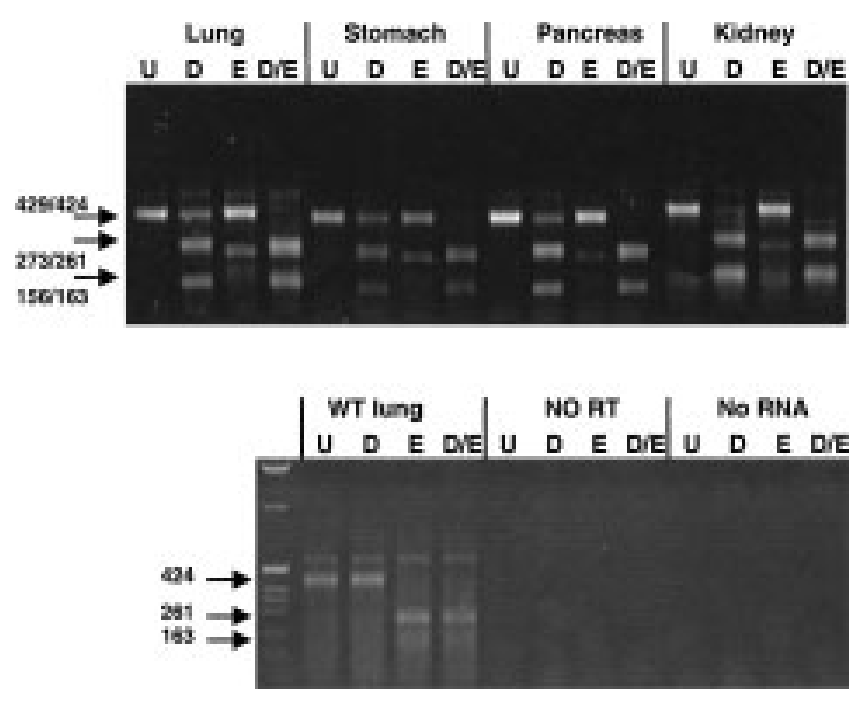

Fig. 6. Detection of the expression of the human MUC1 gene in transgenic mice. Expression of the human MUC1 transgene in transgenic mouse lung, stomach, pancreas and kidney. RT-PCR products derived from the human $M U C 1$ transcript (429 bp) are cleaved with Dra III (D) and those from the murine mucl transcript (424 bp) are cleaved with Eag I (E). $\mathrm{U}$ denotes uncut, D/E double digestion with both enzymes; WT wild type C57BL6 mice. No reverse transcriptase (No RT) controls used transgenic mouse stomach RNA as template.

however, the mechanisms that confer tissue specificity on gene expression in vivo have not been fully elucidated. We identified two novel DHS in the MUC1 gene promoter region at -250 and -750 bp with respect to the major transcriptional start site. These sites were first seen in the HPAF pancreatic adenocarcinoma cell line that expresses high level of endogenous MUC1.

Further evaluation of these DHS showed that the -750 site was present in many cell types, both epithelial and non-epithelial and its intensity did not correlate with levels of MUC1 expression. In contrast, the -250 DHS was only seen in cell types that express relatively high levels of $\mathrm{MUCl}$ and its intensity showed a strong correlation with the abundance of MUCI mRNA. Of particular interest was the observation that $M U C 1$ expression levels were reduced and the -250 DHS became undetectable on Southern blots in cells that model the adenoma to carcinoma transition (AA/Cl to AA/C1/SB10C colonic epithelial cell lines).

Detection of DHS in a human gene in transgenic mice can provide strong support for their importance in vivo. Mice transgenic for the human $M U C 1$ gene carried on a $10.6 \mathrm{~kb}$ Sac II fragment including $5^{\prime}$ and 3' flanking DNA, show a very similar $M U C 1$ expression pattern to that seen for the endogenous $m u c l$ (12). The -750 and -250 DHS were detected in chromatin extracted from certain tissues of these transgenic mice. The relative intensity of the -250 and -750 DHS was not constant, with the -250 DHS being most evident in stomach, though also present in other tissues in which $M U C 1$ transgene expression was high, such as kidney and lung. These data suggest that the mouse contains the necessary transcription factors to interact with the important sequences generating the DHS. It was of interest to compare the -250 and -750 regions of the mouse and human genes for regions of cross-species homology that might be functionally important. A region of about 34 base pairs of high homology mapped to the predicted core of the -750 DHS. A short region of high homology $5^{\prime}$ and a more extended region immediately $3^{\prime}$ to the predicted location of the -250 DHS were also evident.

These regions of high cross-species conservation were re-evaluated to see if they coincided with important regulatory motifs previously identified in the promoter of the MUCl gene. The -750 DHS is close to a predicted half oestrogen response (ER) element and a GC box/Spl site (2) though neither of these have been shown to be functionally important in $M U C 1$ expression.

The -250 DHS corresponds exactly to the location of a region of non-random purine/pyrimidine strand asymmetry (purine/pyrmidine mirror repeat elements-PMR) identified in the $M U C 1$ promoter (M-PMR2) (26), and is proximal to another PMR element (M-PMR3) located at approximately -130 . M-PMR3 is evident in both the human MUC1 and mouse $\mathrm{Mucl}$ genes but M-PMR2 is not conserved. These non-random sequences, which contain perfect or nearly perfect mirror repeat elements, may be associated with the formation of H-DNA (intramolecular triple helical) conformations. The MPMR3 element was shown previously to adopt a relatively uncommon $\mathrm{H}-\mathrm{DNA}$ conformation ( $\mathrm{Hy} 5$ isomer) in vitro (27). M-PMR2 did not exhibit S1 sensitivity (evidence of H-DNA character) in vitro (26) when investigated in a plasmid that contained both M-PMR2 and M-PMR3. However, the MPMR2 element has not been examined in isolation and is predicted to be capable of forming H-DNA structures under conditions of high superhelical tension. The previous experiments that examined MPMR2 and M-PMR3 together (26) were conducted under conditions of standard plasmid superhelical density. The energy from superhelical tension that is required for producing H-DNA conformations was probably absorbed by the formation of H-DNA at the M-PMR3 element in those experiments and may not have been sufficient for formation of a second structure at M-PMR2 (which is shorter and may require more energy). Thus, it remains possible that M-PMR2 could form an H-DNA structure in vivo under conditions of superhelical density that would be predicted to occur in chromatin upstream of a gene undergoing active transcription. The finding of a DHS at -250 that correlates with transcriptional activity supports the hypothesis that altered DNA conformations in this region are associated with transcriptional activity of the $M U C 1$ gene. It is also 


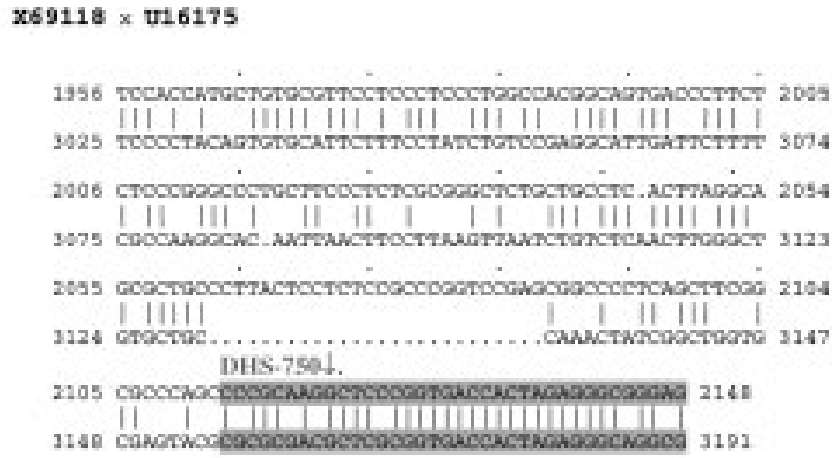

A

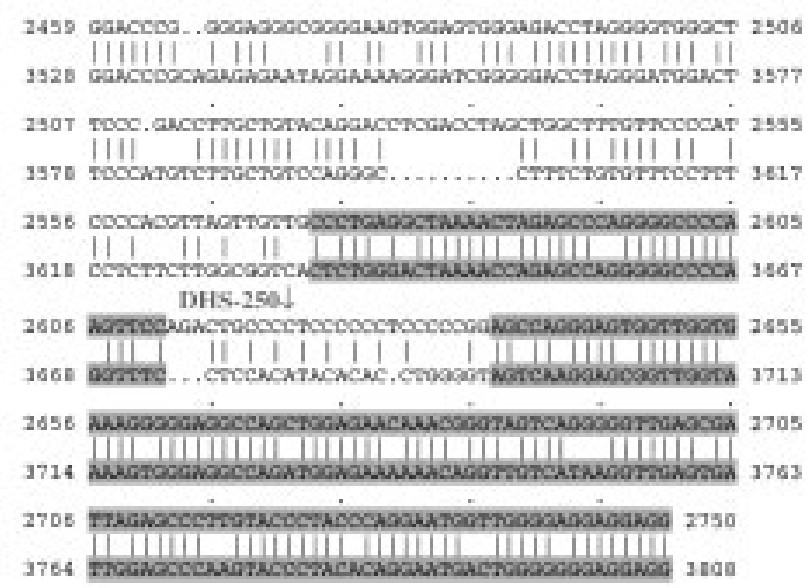

B

Fig. 7. Comparison of the human MUC1 and murine Mucl gene sequences in the region of the -750 and -250 DHS. (A) The -750 DHS. (B) The -250 DHS. Regions of homology between the human (X69118) and murine (U16175) sequences are highlighted.

possible that unknown transcription factors are associated with this element and that these cause the observed DHS. Although the M-PMR3 element was previously shown to not influence transcriptional activity of promoter reporter constructs (28), the MPMR2 element has not been investigated to date. Hence, the role of M-PMR2 in regulation of MUC1 gene expression remains intriguing but unclear.

In summary, we have identified two novel DHS in the MUC1 promoter. One DHS at -750 was detected irrespective of transcriptional activity of the gene. A second DHS at -250 that was associated with transcriptional activity of the gene mapped near previously defined purine/pyrimidine mirror repeat elements that may form intramolecular $\mathrm{H}$ DNA structures. Further elucidation and evaluation of the DNA sequence encompassing the -250 DHS regions is warranted to elucidate the mechanisms of action of potential regulatory elements located within this region of the $M U C 1$ promoter.

\section{Acknowledgments}

We are grateful to Sandra Gendler for transgenic mice and Shinichi Sameshima for help with mouse surgery; also to Christos Paraskeva for the AA/Cl and AA/C1/SB10C cell lines. This work was funded by NIH grant CA79580, the Cystic Fibrosis Trust, UK and a Wellcome Biomedical collaboration grant.

\section{References}

1. Hanisch FG. (2001) O-glycosylation of the mucin type. Biol. Chem. 382: 143-149.

2. Abe M, Kufe D. (1993) Characterization of cis-acting elements regulating transcription of the human DF3 breast carcinoma-associated antigen (MUC1) gene. Proc. Natl. Acad. Sci. USA 90: 282-286.

3. Kovarik A, Peat N, Wilson D, et al. (1993) Analysis of the tissue-specific promoter of the MUCl gene. J. Biol. Chem. 268: 9917-9926.

4. Shirotani K, Taylor Papadimitriou J, Gendler SJ, Irimura T. (1994) Transcriptional regulation of the MUCl mucin gene in colon carcinoma cells by a soluble factor. Identification of a regulatory element. J. Biol. Chem. 269: 15030-15035.

5. Irimura T, McIsaac AM, Carlson D, et al. (1990) Soluble factor in normal tissues that stimulates high-molecular-weight sialoglycoprotein production by human colon carcinoma cells. Cancer Res. 50: 3331-3338.

6. Morris JR, Taylor Papadimitriou J. (2001) The Spl transcription factor regulates cell type-specific transcription of MUC1. DNA Cell Biol. 20: 133-139.

7. Parry G, Li J, Stubbs J, et al. (1992) Studies of Muc-1 mucin expression and polarity in the mouse mammary gland demonstrate developmental regulation of Muc-1 glycosylation and establish the hormonal basis for mRNA expression. J. Cell Sci. 101: 191-199.

8. Gaemers IC, Vos HL, Volders HH, et al. (2001) A statresponsive element in the promoter of the episialin/MUC1 gene is involved in its overexpression in carcinoma cells. J. Biol. Chem. 276: 6191-6199.

9. Scibetta AG, Albanese I, Morris J, et al. (2001) Regulation of MUCl expression in human mammary cell lines by the c-ErbB2 and ras signaling pathways. DNA Cell Biol. 20: 265-274.

10. Williams AC, Harper SJ, Paraskeva C. (1990) Neoplastic transformation of a human colonic epithelial cell line: in vitro evidence for the adenoma to carcinoma sequence. Cancer Res. 50: 4724-4730.

11. Peat N, Gendler SJ, Lalani N, et al. (1992) Tissue-specific expression of a human polymorphic epithelial mucin (MUC1) in transgenic mice. Cancer Res. 52: 1954-1960.

12. Rowse GJ, Tempero RM, VanLith ML, et al. (1998) Tolerance and immunity to MUCl in a human MUCl transgenic murine model. Cancer Res. 58: 315-321.

13. Kim YW, Kern HF, Mullins TD, et al. (1989) Characterization of clones of a human pancreatic adenocarcinoma cell line representing different stages of differentiation. Pancreas. 4: 353-362.

14. Fogh J, Wright WC, Loveless JD. (1977) Absence of HeLa cell contamination in 169 cell lines derived from human tumors. J. Natl. Cancer Inst. 58: 209-2 14.

15. Huet C, Sahuquillo Merino C, et al. (1987) Absorptive and mucus-secreting subclones isolated from a multipotent intestinal cell line (HT-29) provide new models for cell polarity and terminal differentiation. J. Cell Biol. 105: 345-357.

16. Soule HD, Vazguez J, Long A, (1973) A human cell line from a pleural effusion derived from a breast carcinoma. J. Natl. Cancer Inst. 51: 1409-1416.

17. Smith AN, Barth ML, McDowell, et al. (1996) A regulatory element in intron 1 of the cystic fibrosis transmembrane conductance regulator gene. J. Biol. Chem. 271: 9947-9954. 
18. Moulin DS, Manson AL, Nuthall HN, et al. (1999) In vivo analysis of DNase I hypersensitive sites in the human CFTR gene. Mol. Med. 5: 211-223.

19. Becker P, Renkawitz R, Schutz G. (1984) Tissue-specific DNase I hypersensitive sites in the 5'-flanking sequences of the tryptophan oxygenase and the tyrosine aminotransferase genes. EMBO J. 3: 2015-2020.

20. Chirgwin JM, Przybyla AE, MacDonald RJ, Rutter WJ. (1979) Isolation of biologically active ribonucleic acid from sources enriched in ribonuclease. Biochemistry 18: 5294-5299.

21. Chambers JA, Harris A. (1993) Expression of the cystic fibrosis gene and the major pancreatic mucin gene MUCl in human ductal epithelial cells. J. Cell Sci. 107: 413-424.

22. Higgs DR, Wood WG, Jarman AP, et al. (1990) A major positive regulatory region located far upstream of the human alpha-globin gene locus. Genes Dev. 4: 1588-1601.

23. Ogata S, Uehara H, Chen A, Itzkowitz S. (1992) Mucin gene expression in colonic tissues and cell lines. Cancer Res. 52: 5971-5978.
24. Hollingsworth MA, Strawhecker JM, Caffrey TC, Mack DR. (1994) Expression of MUC1 MUC2 MUC3 and MUC4 mucin mRNAs in human pancreatic and intestinal tumor cell lines. Int. J. Cancer. 57: 198-203.

25. Vavasseur F, Dole K, Yang J, et al. (1994) O-glycan biosynthesis in human colorectal adenoma cells during progression to cancer. Eur. J. Biochem. 222: 415-424.

26. Hollingsworth MA, Closken C, Harris A, et al. (1994) A nuclear factor that binds purine-rich single-stranded oligonucleotides derived from S1-sensitive elements upstream of the CFTR gene and the MUC1 gene. Nucleic Acids Res. 22: 11381146.

27. Nelson KL, Becker NA, Pahwa GS, et al. (1996) Potential for H-DNA in the Human MUC1 mucin gene promoter. J. Biol. Chem. 271: 18061-18067.

28. Pahwa GS, Maher LJ, Hollingsworth MA. (1996) A potential H-DNA element in the MUC1 promoter does not influence transcription. J. Biol. Chem. 271: 26543-26546. 